\title{
Standardization of Anatomic Liver Resection Based on Laennec's Capsule
}

\author{
Atsushi Sugioka, Yutaro Kato, Yoshinao Tanahashi, Jun-ichi Yoshikawa, Gozo Kiguchi, \\ Masayuki Kojima, Akira Yasuda, Sanae Nakajima, Ichiro Uyama
}

Department of Surgery, Fujita Health University, Aichi, Japan

\section{ABSTRACT}

We proposed a novel comprehensive surgical anatomy of the liver based on Laennec's capsule in 2017, which contributed to the standardization of extrahepatic Glissonean pedicle isolation-the optimal method for Glissonean pedicle isolation without parenchymal destruction. Thereafter, the scope of our concept included an extension to the hepatic vein and the plate system to establish the surgical technique of the anatomic liver resection that consisted of extrahepatic Glissonean pedicle isolation, exposure of the landmark hepatic vein, and parenchymal reresection to the optimum amount. The accumulated histological evidence supported our concept, employing elastic fiber staining as a means of visualization. For the standardization of extrahepatic Glissonean pedicle isolation, it was necessary to accurately approach the six gates marked by four anatomical landmarks to enter a gap between the Glissonean pedicle and the Laennec's capsule that covers the liver parenchyma. The cystic plate cholecystectomy and division of the anchors facilitate the procedure. For the standardization of the exposure of the hepatic vein-another essential procedure for the anatomic liver resection - it was important to identify the two layers of Laennec's capsule around the hepatic vein and preserve them by appropriately taking one of the following three approaches: the outer-Laennec, inter-Laennec, and inner-Laennec. The hepatic vein root at first one-way dissection in cranio-caudal direction was quite useful in exposing the hepatic vein. Surgical techniques based on Laennec's capsule led to all kinds of standardized anatomic liver resection, contributing to establishing a safe standard within hepatobiliary surgery, especially in minimally invasive surgical procedures.

Key words: anatomic liver resection, Glissonean pedicle, plate system, hepatic vein, Laennec's capsule

\section{INTRODUCTION}

Liver resection remains a challenging procedure, with common complications including bleeding, bile leakage, and liver failure. Additionally, the procedure is yet to be standardized, primarily because our understanding of detailed liver anatomy to date has been incomplete. Laennec's capsule is a proper membrane of the liver described by RTH Laennec in 1802 (1), which

\author{
Corresponding author: \\ Atsushi Sugioka, M.D. Ph.D. \\ Department of Surgery \\ Fujita Health University \\ 1-98 Dengakugakubo \\ Kutsukake-cho, Toyoake \\ Aichi, 470-1192, Japan \\ Tel: +81-562-93-9254; \\ Fax: +81-562-93-7060 \\ E-mail: sugioka@fujita-hu.ac.jp
}

\section{Abbreviations: \\ HV: hepatic vein IVC: inferior vena cava LHV: left hepatic vein MHV: middle hepatic vein RHV: right hepatic vein SS: subserosal layer MP: proper muscle layer $\mathrm{M}$ : mucosal layer}


covers the entire liver surface, and also the intrahepatic liver parenchyma surrounding the Glissonean pedicles and the hepatic veins (2). This knowledge is essential for understanding the intricacies of liver anatomy that will likely lead to the standardization of a variety of hepatobiliary surgeries. However, its acute significance has been ignored for over 200 years and was called a not so important envelope by Couinaud (2). The availability of minimally invasive procedures is increasing and there is a high demand for standardized practices to be defined in the context of liver procedures. As a result, precise liver anatomy is gaining attention as the safest course of action and, accordingly, the importance of Laennec's capsule has been carefully reviewed $(3,4)$. Nonetheless, as it was lacking practicability, it was insufficient for standardization. In 2017, we proposed a novel comprehensive surgical anatomy of the liver based on Laennec's capsule and the standardized extrahepatic Glissonean pedicle isolation (5). Glissonean pedicle isolation is the optimal pedicle isolation technique due to its lack of parenchymal destruction, proposed by Couinaud (6) and first performed by Takasaki (7). Thereafter, the scope of this concept based on Laennec's capsule has rapidly extended to the hepatic vein, and to the plate system that contributed to establish various anatomic liver resections with the assistance of histological evidence (8). In this study, we report recent elucidations surrounding liver anatomy in the context of Laennec's capsule and the resulting detailed surgical techniques for anatomic liver resections.

\section{SURGICAL TECHNIQUES}

\section{Standardization of surgical techniques for the anatomic liver resection}

We proposed a novel surgical anatomy of the liver based on Laennec's capsule as shown in figure 1, which prioritizes the entirety of Laennec's capsule (red line) (5). Hayashi had already histologically identified the portion of Laennec's capsule that surrounded the Glissonean pedicle and the hepatic vein toward the periphery via the definite index of elastic fiber staining (8). Based on this concept, we standardized the anatomic liver resection, which is a fundamental procedure consisting of extrahepatic Glissonean pedicle-first isolation, the hepatic vein root at first oneway resection in the cranio-caudal direction, and then obtained a necessary and sufficient parenchymal resection. In our study, we described these surgical techniques in detail.

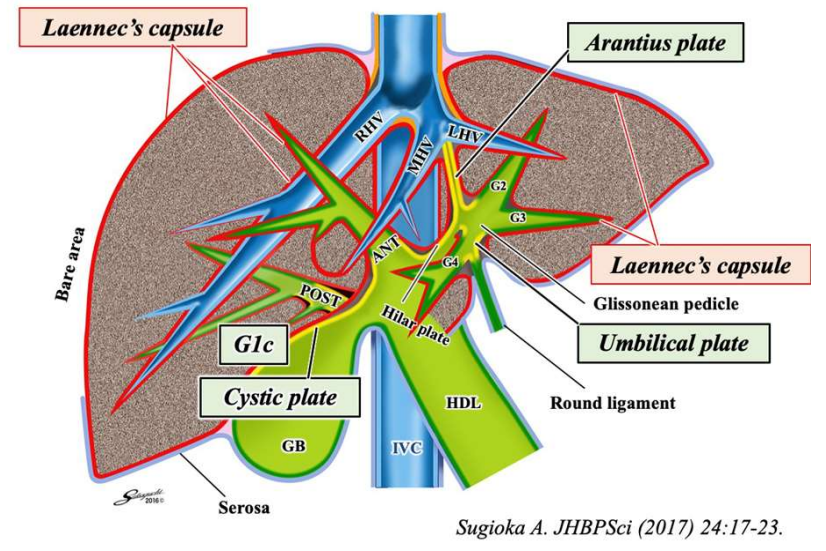

Figure 1 - The schema of Laennec's capsule (based on Sugioka 2017)

This schema shows Laennec's capsule (red line). It covers not only the entire surface of the liver parenchyma including the bare area but also the intrahepatic liver parenchyma surrounding the Glissonean pedicles and the hepatic veins.

\section{Extrahepatic Glissonean pedicle isolation - first approach based on Laennec's capsule}

\section{Anatomical considerations on extrahepatic Glissonean pedicle isolation}

\section{The Glissonean pedicle}

The Glissonean pedicle was described by Glisson in 1654 (9), recognized by the membranous structure around the pedicle body (figure $2 a$ ). However, since it had been misunderstood as the alleged 'Glissonean capsule' for many years, a holistic understanding of extrahepatic Glissonean pedicle isolation was hampered. This is because destruction of the parenchyma is inevitable during isolation if the Glissonean pedicle is directly in contact with the liver parenchyma. However, Hayashi clearly demonstrated that the alleged 'Glissonean capsule' belongs to the liver parenchyma as Laennec's capsule (8). Further, there is a gap between the Glissonean pedicle and Laennec's capsule, covering the liver parenchyma. As shown in figure $2 b$, the grey spaces (indicated by arrows) provide theoretical background to extrahepatic Glissonean pedicle isolation (5).

\section{The plate system (figure 3 )}

The plate system was first described by Couinaud $(10,11)$, commonly misunderstood as the separable membranous structure outside the Glissonean pedicle at the hilum. However, such a structure could not be found histologically, but a thickened part of the Glissonean pedicle comprising collagen-rich connective tissue containing aberrant biliary glands and hyper- 

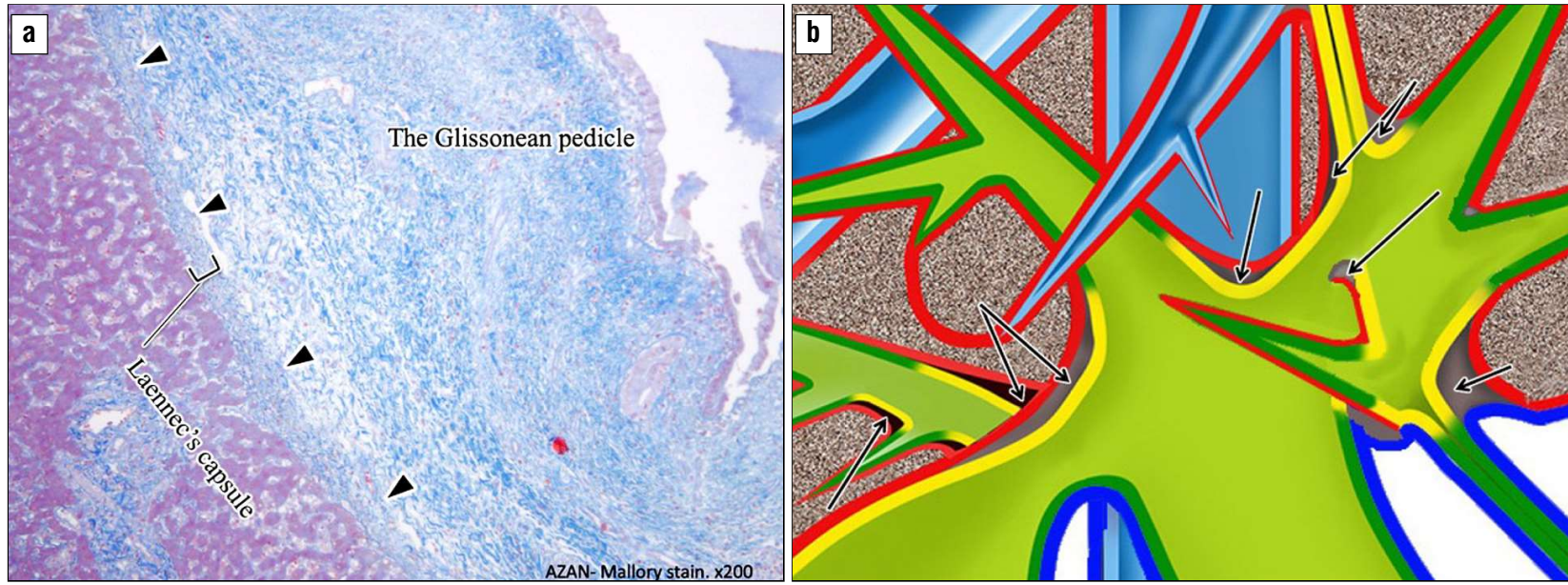

Figure 2 - Theoretical background of extrahepatic Glissonean pedicle isolation

a. Histological findings of the Glissonean pedicle (Azan-Mallory staining, x200). A Glissonean pedicle was surrounded by the dense fibrous layer which had been long misunderstood as 'Glissonean capsule'. However, Hayashi revealed the layer was Laennec's capsule belonging to the liver parenchyma. Gaps are observed inside the fibrous layer (arrow heads).

b. Schema of gaps between the Glissonean pedicle and Laennec's capsule covering the liver parenchyma. There exist gaps (Gray spaces indicated by arrows) between the Glissonean pedicles and Laennec's capsule.

trophic lymphoid tissues was detected, as shown in figure $3 a$ and $3 b$. Therefore, the plate system should be included as a part of the Glissonean pedicle (figure 3c, $3 d$ ). Couinaud already recognized the Glissonean pedicle as a vascular sheath without a capsule and the hilar plate as a thickened solid part of the pedicle surface (2). This provides another reason explaining why the gap between the surface of the Glissonean pedicle (the plate system) and Laennec's capsule can be entered.

\section{Anchors (figure 4)}

Anchors are the thin, cord-like structures found at the orifices of the Glissonean pedicles that connect the pedicles with Laennec's capsule, as shown in figure $4 a$ (their distributions shown in figure $4 b$ ). Owing to the

\section{Figure 3 - The plate system}

a. The vertical-sectional surface at the main portal fissure of the resected specimen including the hepatic hilum. GB: gall bladder.

b. Histological findings of the hepatic hilum (hematoxylin-eosin staining, x100). The collagen-rich connective tissue containing aberrant biliary glands and hypertrophic lymphoid tissues was found but no membranous structure was found.

c. Schema of the plate system. The plate system should be regarded as a thickened part of the Glissonean pedicle.

d. Detailed schema of the plate system

This image shows the whole plate system.
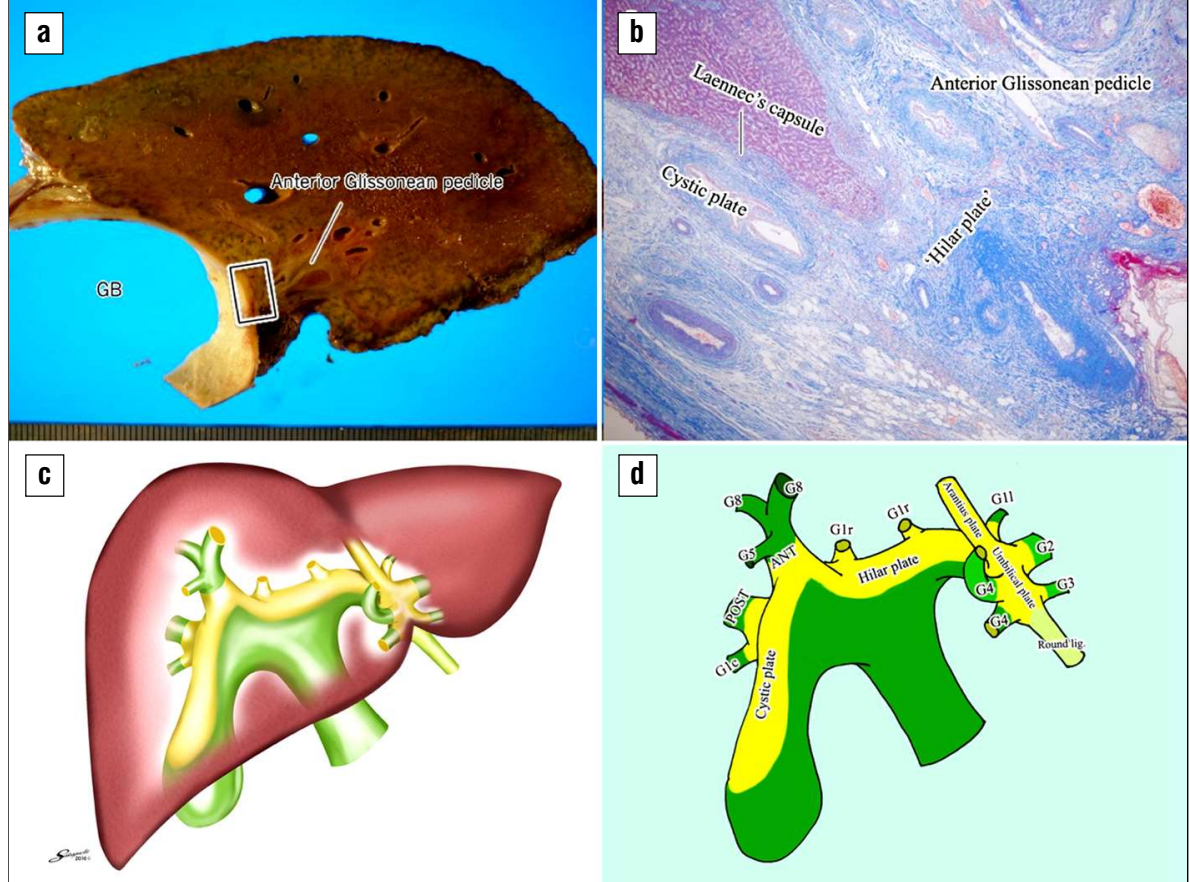


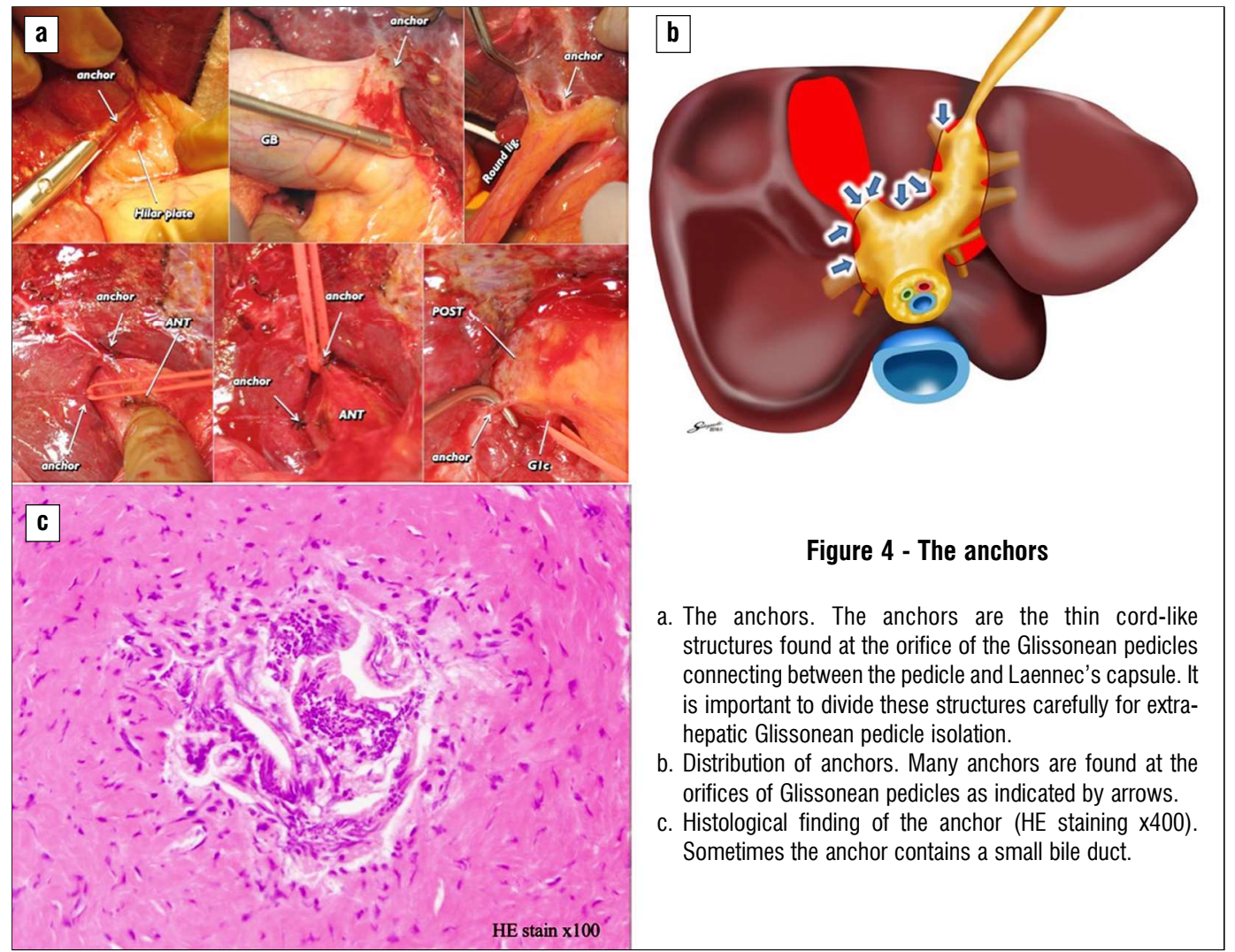

magnified view of the minimally invasive surgery, we noticed them and recognized the significance of extrahepatic Glissonean pedicle isolation because they sometimes contain small bile ducts that cause bile leakage, as shown in figure $4 c$.

\section{Surgical techniques for extrahepatic Glissonean pedicle isolation (video1)}

The entire surgical technique of extrahepatic Glissonean pedicles based on Laennec's capsule is demonstrated in video 1.

\section{Approach to the four anatomical landmarks and the six gates (figure 5)}

Extrahepatic Glissonean pedicle isolation is an important procedure that enables surgeons to control inflow and confirm the target area prior to the parenchymal dissection. However, the technique had not been standardized until recently because of the difficulty associated with isolating specific pedicles extrahepatically. According to our novel anatomy based on Laennec's capsule, we found that only the six gates indicated by the four anatomical landmarks allow us to approach the gap between the Glissonean pedicle and Laennec's capsule and to isolate the target pedicle extrahepatically, as shown in figure $5 a$ (5). The four anatomical landmarks were defined as follows: the Arantius plate, the umbilical plate, the cystic plate, and the Glissonean pedicle of the caudate process (G1c). They indicate the six gates and connecting two of these gates enables us to isolate each secondorder pedicle (figure $5 b, 5 c$ ). The validity of extrahepatic Glissonean pedicle isolation was clearly demonstrated histologically by Morimoto with his precise laparoscopic technique using the cadaver model. At first, we developed several conceptual procedures based on Laennec's capsule (procedures included were those such as segmentectomy 8 and isolated total caudate) using extrahepatic Glissonean pedicle-first isolation in both open and minimally invasive approaches as described by Kato. Even third-order or more peripheral branches of the Glissonean pedicles can be pulled out and be safely isolated extrahepatically at the hilum (figure $5 d$ ). Currently, extrahepatic Glissonean pedicle isolation without parenchymal destruction has become much more imperative with the advent of ICG fluorescence.

\section{Division of anchors}

To safely isolate the extrahepatic Glissonean pedicles, careful division of the anchors was also 


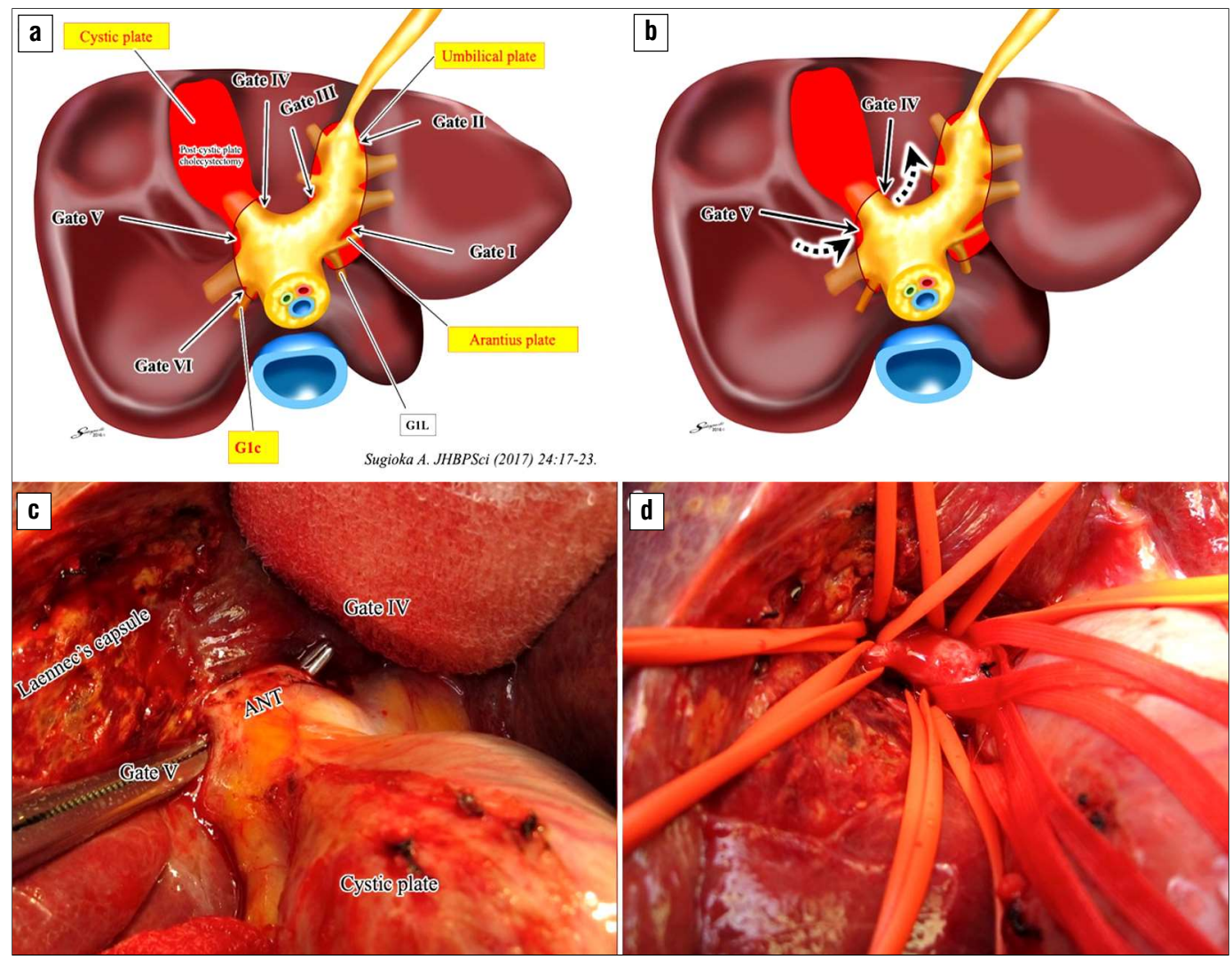

Figure 5 - Standardization of extrahepatic Glissonean pedicle isolation using six gates indicated by four anatomical landmarks

a. Schema of the six gates indicated by the four anatomical landmarks (based on Sugioka 2017). Only the six gates indicated by the four anatomical landmarks allows us to enter a gap between the Glissonean pedicle and Laennec's capsule covering the liver parenchyma. Anatomical landmarks are the Arantius plate, Umbilical plate, Cystic plate, and Glissonean pedicle of the caudate process (G1c).

b. Schema of anterior Glissonean pedicle isolation between the Gate IV and V. By connecting the Gate IV and V, the anterior Glissonean pedicle can be isolated extrahepatically.

c. Intraoperative photograph of anterior Glissonean pedicle isolation

d. Intraoperative photograph of peripheral Glissonean pedicles isolation. Accurate approach to the Gates enables us to isolate further peripheral Glissonean pedicles extrahepatically by pulling them out towards the hepatic hilum safely.

essential to confirm the contour of the target pedicle and prevent bile leakage as they sometimes contain small bile ducts (figure $4 c$ ).

\section{Cystic plate cholecystectomy (figure 6)}

We developed a novel cholecystectomy technique called the 'cystic plate cholecystectomy' based on our anatomical appreciation of Laennec's capsule. This technique revealed that the layer of the gall bladder shared with the liver bed is composed of six layers instead of the conventionally taught three layers (figure 6a-i). Accordingly, we proposed this procedure to standardize extrahepatic anterior Glissonean pedicle isolation (Figure 6a-ii). The gall bladder along with the cystic plate was removed from Laennec's capsule, and this procedure accurately led us to the layer of the anterior Glissonean pedicle (figure $6 b$, $6 c)$. The histo-logical findings were confirmed the structure as shown in figure $6 d$ and $6 e$. Furthermore, upward blunt dissection from the boundary between the neck and body was enough to perform this procedure, indicating that there was a so called 'grain of the turf', whereas the grain seems inverted from the neck to the hilum and naturally led us to the anterior Glissonean pedicle as shown in Video 1.

\section{Exposure of the hepatic veins (video 2)}

The exposure of the hepatic vein was another essential procedure for anatomic liver resection requiring precise anatomical understanding. Detailed procedures are demonstrated in Video 2. 


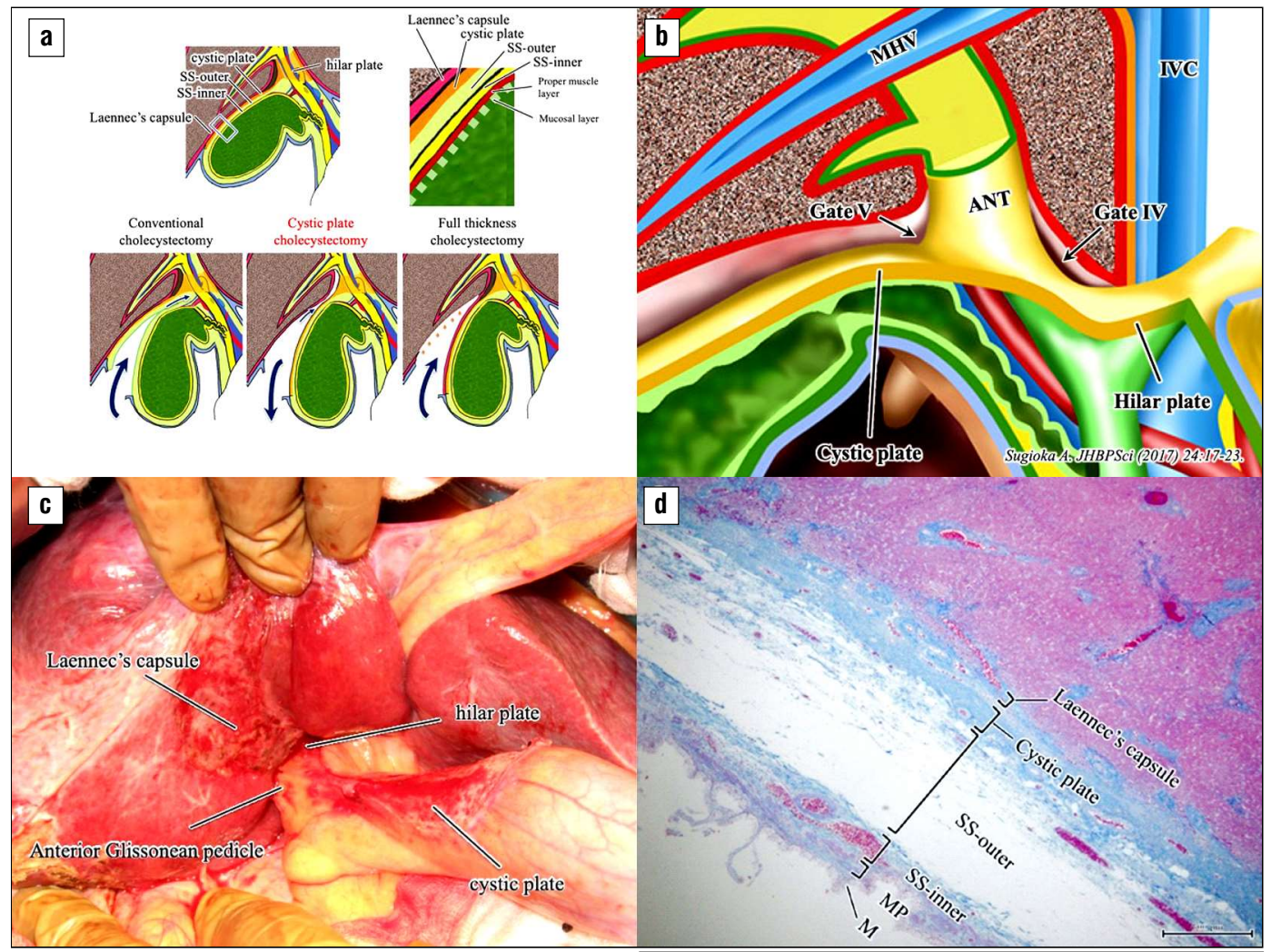

Figure 6 - Cystic plate cholecystectomy. A novel procedure of cholecystectomy named 'cystic plate cholecystectomy' was developed for standardization of the anterior Glissonean pedicle isolation.

a. The schema of the layers of the gall bladder and liver bed based on Laennec's capsule and comparison to another cholecystectomies

i. The layer of the gall bladder and liver bed is consisted of six layers instead of the conventional concept of three layers.

ii. In the conventional cholecystectomy, the SS-outer layer was dissected which is continuous to the Glissonean sheath Consequently, potential danger of biliary and vascular injury should be recognized.

iii. As shown, the cystic plate cholecystectomy is an optimal procedure to approach the anterior Glissonean pedicle.

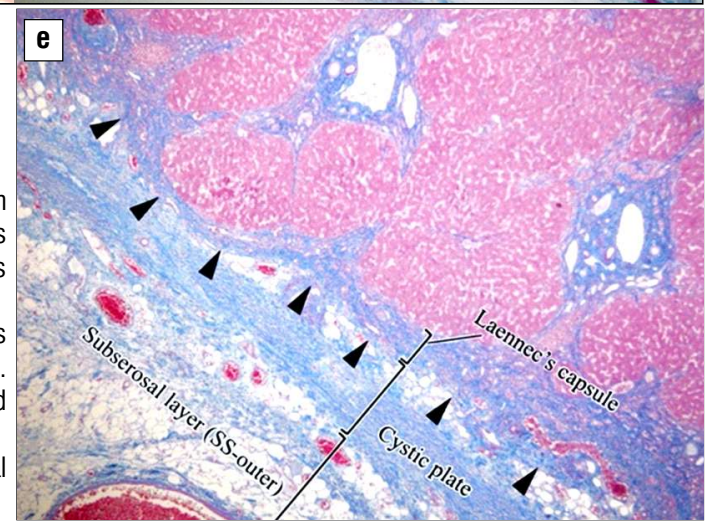

iv. The full thickness cholecystectomy, in which Laennec's capsule is peeled off from the liver parenchyma with some bleeding, is a different procedure from the cystic plate cholecystectomy.

b. The schema of the cystic plate cholecystectomy. It is clearly demonstrated that this procedure leads us to the surface of the anterior Glissonean pedicle.

c. The photograph after the cystic plate cholecystectomy. The cystic plate is clearly visualized which continues to the hilar plate and the anterior Glissonean pedicles, whereas the liver bed is covered by Laennec's capsule

d. The histological finding of the gall bladder shared with the liver bed (Azan Mallory stain, x 100). The histological finding revealed the six layers of the gall bladder shared with the liver bed. (SS; subserosal layer, MP; proper muscle layer, M: mucosal layer).

e. The high-magnification image of the histological finding (Azan Mallory stain, x 400). The high-magnification image of the histological finding clearly demonstrated the detailed structures; Laennec's capsule, the cystic plate, and the SS-outer layer.

\section{Anatomical considerations for hepatic vein exposure}

a. The hepatic vein

The hepatic vein is a unique structure that runs independently from the inflow of the Glissonean pedicle. The hepatic veins are the important landmarks of the hepatic segments and their exposure was an essential technique for anatomic liver resection (13).
However, their exposure is a difficult procedure causing massive bleeding and standardization has not been achieved due to a lack of anatomical understanding. Laennec (1) and Couinaud (2) had already described Laennec's capsule around the root of the main hepatic veins and Hayashi placed it at the peripheral hepatic veins (8). Recently, Kiguchi histologically revealed that the hepatic veins are surrounded by two layers of Laennec's capsule: one from the proper membrane of 
the liver (the hepatic Laennec's capsule) and the other derived from the pericardium (the cardiac Laennec's capsule). Based on these findings, he also proposed novel approaches to safely expose the hepatic veins $(13,14)$.

\section{Laennec-web (figure 7)}

The Laennec-web was the cobweb-like structure composed of a branched thread-like composition, often encountered during liver resection, especially when exposing the hepatic veins (figure 7). It is likely that they came from the peripheral Glissonean pedicles and connected to the preserved Laennec's capsule on the hepatic veins. We speculated that these structures represent the remnant of Laennec's capsule after all Glissonean pedicles were delivered to the hepatic lobules. These structures might shed light on the embryologic origin of Laennec's capsule.

\section{Surgical techniques for hepatic vein exposure (Video 2)}

The exposure of the landmark hepatic vein was another essential procedure for anatomic liver resection (12). However, the procedure was demanding and even dangerous with bleeding when performed using the conventional method of parenchymal dissection in caudo-cranial direction. The techniques were comprehensively expressed as 'the hepatic vein root-at first one-way dissection in the cranio-caudal direction with an appropriate selection of the layer'.

\section{Hepatic vein root-at first cranio-caudal dissection (video 2)}

The HV root-at first parenchymal dissection in the cranio-caudal direction exposing the hepatic veins towards the periphery and a parenchymal removal in necessary and sufficient was achieved.

Selection of dissecting layers of Laennec's capsule around the root of the hepatic vein and IVC (figure 8)

Since Laennec's capsule surrounding the hepatic vein was composed of two layers, we could select the appropriate layer for dissection to preserve one or two layers of Laennec's capsule. Based on this concept, Kiguchi proposed three types of approaches to expose the major hepatic veins, namely, outer-Laennec (figure 8a), inter-Laennec (figure 8b), and innerLaennec (Figure 8c). These approaches should be selected according to the kinds of hepatic veins and proximity to the tumor. Histological findings and

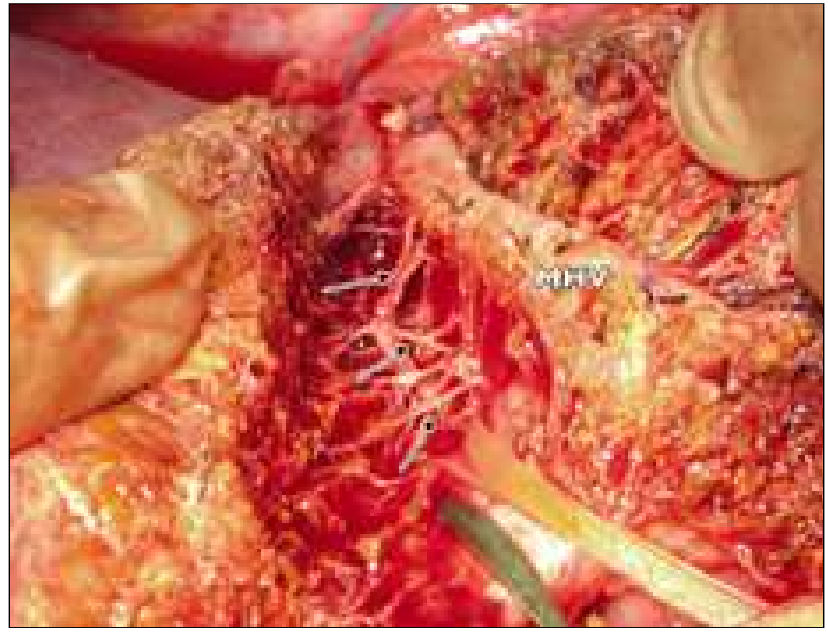

Figure 7 - Laennec-web. The Laennec-web is the cobweb-like structure composed of a branched thread-like composition often encountered during liver resection especially when exposing the hepatic veins.

procedures were clearly demonstrated by Monden on this issue. The outer-Laennec approach (figure 8a) demonstrated the exposure of the hepatic veins, having preserved both layers of Laennec's capsule that cover it. This information is especially useful for central hepatectomies such as the central bisectionectomy, anterior sectionectomy, medial sectionectomy, and segmentectomy 8 , for which both major hepatic veins must be exposed. The root-at first cranio-caudal dissection is also essential. The inter-Laennec approach (figure $8 b$ ) means entering the inter-Laennec space preserving only the cardiac Laennec's capsule around the hepatic vein. This procedure is useful for right and left hemihepatectomy, right posterior sectionectomy (13), and segmentectomy 7 (14). The root-at first cranio-caudal dissection and the dorsal parenchymal approach is useful for right-side liver resection in the semi-prone position. On the contrary, the inner-Laennec approach (figure $8 c$ ) involves exposing the adventitia of the hepatic vein with peeling off the both the layers, making the vein wall fragile. The conventional parenchymal dissection in caudo-cranial direction tends to fall into the inner-Laennec approach and the vein wall is easily split. Although expert hands may deal with proficiently, such a potentially dangerous approach should be avoided by selecting the outer- or inter-Laennec approach as much as possible.

\section{One-way parenchymal resection (figure 9)}

One-way parenchymal resection involves the comprehensive concept to standardize the anatomic liver resection including extrahepatic Glissonean pedicle isolation followed by the one-way parenchymal 


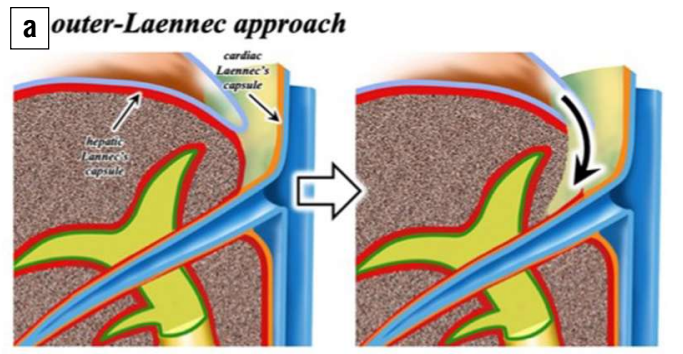

b inter-Laennec approach

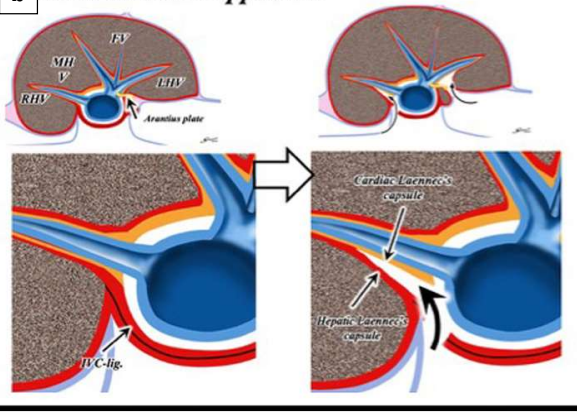

a. The outer-Laennec approach means the exposure of the hepatic veins preserving the both two layers of Laennec's capsule around the hepatic veins that is useful especially for central hepatectomies.

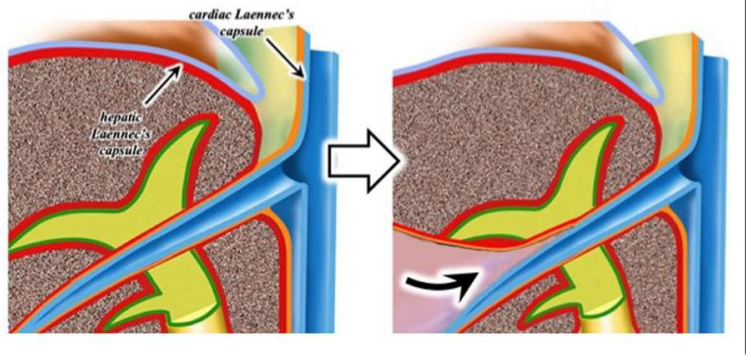

b. The inter-Laennec approach means entering the interLaennec space preserving only the cardiac Laennec's capsule around the hepatic vein.

c. the inner-Laennec approach means exposing the adventitia of the hepatic vein with peeling off the both two layers and the vein wall becomes fragile. This potentially dangerous approach should be avoided by selecting the outer- or inter-Laennec approach as much as possible.

Figure 8 - Selection of dissecting layers of Laennec's capsule around the root of the hepatic vein and IVC Laennec's capsule surrounding the hepatic vein is composed of two layers, we can select the appropriate layer of dissection to preserve one or two layers.
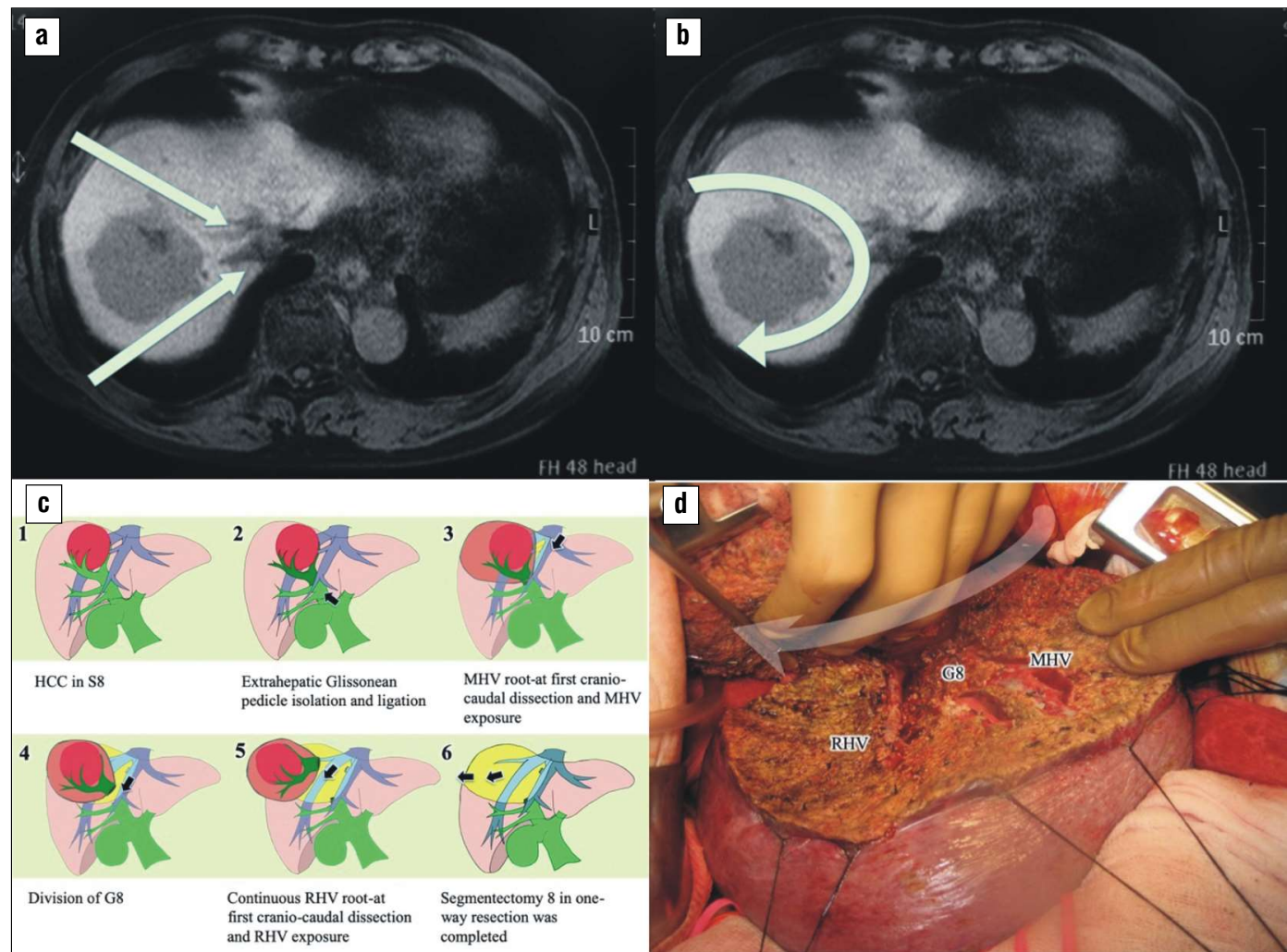

Figure 9 - One-way resection

a. Conventional two-way resection for central hepatectomy

b. One-way resection for central hepatectomy

c. Schema of segmentectomy 8 in one-way resection in cranio-caudal direction

d. Intraoperative photograph during segmentectomy 8 in one-way resection. The MHV and RHV were clearly exposed and flat resection plane was obtained. It is suggested that the parenchymal dissection was performed along the so called 'intersegmental plane' 
dissection using the hepatic vein root-at first parenchymal dissection in the cranio-caudal direction exposing the hepatic veins towards the periphery, and a parenchymal removal in necessary and was achieved sufficiently (figure 9a, 9b). This concept could be applied to standardize all anatomic liver resections as the universal procedure and can be useful, especially for the central hepatectomies during which we conventionally dissect the liver parenchyma by forming the two planes separately in caudo-cranial direction and exposing two major hepatic veins using the innerLaennec approach frequently falling into a fragile surface. Thus, such central hepatectomies were complicated and dangerous, whereas the one-way resection enabled us to perform safe and simple anatomic resections without changing the operative field to obtain the so called 'intersegmental plane' as shown in figure $9 c$ and $9 d$.

\section{DISCUSSION}

Liver resection is still a difficult surgery due to lack of standardization, mainly due to a lack of understanding of the precise liver anatomy. Although most experienced liver surgeons are familiar with extrahepatic Glissonean pedicle isolation, the lack of theoretical background had been preventing the standardization of a procedure. Our novel concept provides a clear background and contributes to standardize extrahepatic Glissonean pedicle isolation. Subsequently, the significance of the concept was gradually recognized, especially due to a recent rapid spread of minimally invasive liver resection. For safety, it was ideal to extrahepatically control target Glissonean pedicles at first to identify the accurate target area. In this paper, we described the extended concept of Laennec's capsule from the Glissonean pedicle to the hepatic vein and the plate system. The comprehensive concept of Laennec's capsule contributed to clarifying the precise liver anatomy and for standardizing safe anatomic liver resections. Extrahepatic Glissonean pedicle isolation has been standardized by the six gates, indicated by the four anatomical landmarks that enabled us to enter the gap between the Glissonean pedicle and Laennec's capsule covering the liver parenchyma. Division of anchors and cystic plate cholecystectomy were also useful. The importance of extrahepatic Glissonean pedicle isolation without parenchymal destruction has increased with the recent advent of ICG fluorescence. The exposure of the landmark veins has also been standardized by the hepatic vein root-at first one-way parenchymal dissection in the cranio-caudal direction with the appropriate selection of the layer-the outer-Laennec, interLaennec, and inner-Laennec approach. Owing to these procedures, all kinds of anatomic liver resections are expected to become standard procedures. These surgical techniques were at first only conceptual but now they are standard procedures that suggested the validity of our concept. When we proposed our concept, there were only a few histological evidences on Laennec's capsule. However, the gradually accumulating histological evidences facilitated the standardization of anatomic liver resection.

There were some limitations. First, the existence and origin of Laennec's capsule around the hepatic veins are still controversial due to few histological evidence (13-16), and consensus has not been reached on the terminology of 'cardiac Laennec's capsule'. Second, the reason why Laennec's capsule has such strange structure, which continues from the Glissonean pedicles to the hepatic veins, is unclear and would require embryological study. Therefore, supporting evidence by the basic medicines is mandatory to generalize and diffuse the right skills. The comprehensive surgical anatomy of the liver once fully established would render all kinds of hepatobiliary surgery as safe and standard procedures in the near future.

\section{CONCLUSION}

The detailed liver anatomy that focuses on Laennec's capsule has the capacity to perpetuate a standardization of anatomic liver resection. In future, all kinds of hepatobiliary surgery might be possible based on Laennec's capsule, providing safe standardized procedures.

\section{Contribution of authors}

AS and YK equally contributed to this article.

\section{Conflicts of interest and source of funding}

There are no conflicts of interest to declare. This study received no specific grant from any funding agency.

\section{REFERENCES}

1. Laennec RTH. Lettre sur des Tuniques qui enveloppent certains Viscères, et fournissentdes gaines membraneuses à leurs vaisseaux. Journ De Méd Chir Et Pharm Vendémiaire an XI; 1802. p. 539-575, et Germinal an XI; 1802. p. 73-89.

2. Couinaud C. The vasculo-biliary sheath. Surgical anatomy of the liver revisited. Paris: pers Ed; 1989. p. 29-39.

3. Takasaki K, Yamamoto M. Surgical anatomy of the liver in the 
Glissonean pedicle approach: what we need to know. In: Madoff DC Makuuchi M, Nagino M, Vauthey JN, eds. Venous Embolization of the Liver: Radiologic and Surgical Practice. Springer: 2011. p.23-28.

4. Yamamoto M, Katagiri S, Ariizumi S, Kotera Y, Takahashi Y, Egawa H. Tips for anatomical hepatectomy for hepatocellular carcinoma by the Glissonean pedicle approach (with videos). J Hepatobiliary Pancreat Sci. 2014:21:53-56.

5. Sugioka A, Kato Y, Tanahashi Y. Systematic extrahepatic Glissonean pedicle isolation for anatomical liver resection based on Laennec's capsule: proposal of a novel comprehensive surgical anatomy of the liver. J Hepatobiliary Pancreat Sci. 2017; 24(1):17-23

6. Couinaud C. A simplified method for controlled left hepatectomy. Surgery. 1985; 97:358-61.

7. Takasaki K, Kobayashi S, Tanaka S. Muto H, Watayo T, Saito A, et al Newly developed systematized hepatectomy by Glissonean pedicle transection method. Syujutsu. 1986; 40:7-14. (in Japanese)

8. Hayashi S, Murakami G, Onitsuka A, Itoh M, Nakano T, Fukuzawa Y. Connective tissue configuration in the human liver hilar region with special reference to the liver capsule and vascular sheath. J Hepatobiliary Pancreat Surg. 2008,15:640-647.

9. Glisson F. Anatomia hepatis. London: 0. Pullein; 1654.

10. Couinaud C. Les enveloppes vasculo-biliaires du foie ou capsule de
Glisson. Leur intérêt dans la chirurgie vésiculaire, les résctions hépatiques et l'abord du hile du foie. Lyon Chir. 1954; 49:589-607.

11. Couinaud C. Le foie; etudes anatomiques et chirurgicales. Paris: Masson; 1957:221-222.

12. Honda G, Iwanaga T, Kurata M, Watanabe F, Satoh H, Iwasaki K. The critical view of safety in laparoscopic cholecystectomy is optimized by exposing the inner layer of the subserosal layer. J Hepatobiliary Pancreat Surg. 2009;16: 445-449.

13. Kishi $Y$ Hasegawa $K$, Kaneko J, Aoki T, Beck $Y$, Sugawara $Y$, Makuuchi M, Kokudo N. Resection of segment VIII for hepatocellular carcinoma. Br J Surg. 2012; 99:1105-12.

14. Kiguchi G, Sugioka A, Kato Y, Uyama I. Use of the inter-Laennec approach for laparoscopic anatomical right posterior sectionectomy in semi-prone position. Surg Oncol. 2019; 29:140-141.

15. Kiguchi G, Sugioka A, Kato Y, Uyama I. Laparoscopic S7 segmentectomy using the inter-Laennec approach for hepatocellular carcinoma near the right hepatic vein. Surg Oncol. 2019; 31:132-134.

16. Shirata C, Hasegawa K, Halkic N, Kokudo N. Laennec's capsule does not exist around the peripheral hepatic veins. J Hepatobiliary Pancreat Sci. 2019; 26(10):E13.

17. Sugioka A. Re: Laennec's capsule does not exist around the peripheral hepatic veins. J Hepatobiliary Pancreat Sci. 2019; 26(10): E14. 